\title{
A Clinical Commentary on the Articles "Strategies for Tissue Engineering Cardiac Constructs to Affect Functional Repair Following Myocardial Infarction" and "Stem Cell-Based Cardiac Tissue Engineering"
}

\author{
Repairing, Reprogramming, and Renewing: The Promise of Myocardial Cytotherapeutics
}

\author{
Navin K. Kapur
}

Received: 2 June 2011 / Accepted: 10 June 2011 / Published online: 11 August 2011

(C) Springer Science+Business Media, LLC 2011

Heart failure is a major cause of global morbidity and mortality. Over 24 million individuals worldwide have been diagnosed with heart failure with the number of affected individuals projected to approach 20 million by the year 2020 in the USA alone [1-3]. Historically, therapies for heart failure have focused on modulating hemodynamic loading conditions to ameliorate symptoms with salutary effects on cardiac remodeling that improve survival. Surgical advances have also led to improved outcomes after cardiac transplantation; however, the limited number of donor hearts available has driven future therapies into two arenas: biomechanical and biomolecular. The promise of mechanical support devices (MSDs) for end-stage heart failure is being realized; however, this option remains costly and invasive while demonstrating only a limited long-term survival benefit [4]. Furthermore, the long-term impact of device-based ventriculo-vascular coupling remains poorly understood as these devices have altered the overall pattern of circulation to non-pulsatile flow in recipients of MSDs. Over the past two decades, cell-based approaches for heart failure have emerged on a wave of scientific evidence supporting the possibility of generating cells that communicate, conduct, and contract.

This article is a commentary on the following articles: $10.1007 /$ s12265-011-9303-1 and 10.1007/s12265-011-9307-x

\section{N. K. Kapur $(\bowtie)$}

Molecular Cardiology Research Institute, Division of Cardiology,

Tufts Medical Center,

800 Washington Street, Box \# 80, Boston, MA 02111, USA

e-mail: Nkapur@tuftsmedicalcenter.org
Two reviews in this edition of the Journal of Cardiovascular Translational Research provide insight into the complexity of cytotherapy from the biomedical engineering perspective. Nunes et al. provide a comprehensive overview of stem cell-based cardiac tissue engineering. Their review highlights the challenges of cell injection and tissue replacement strategies, and then examines the role of embryonic and adult stem cells. As highlighted by their review, one of the major issues facing scientists and physicians in this arena is the ability to accurately gauge improvement in cardiac function after cell therapy. Left ventricular ejection fraction (LVEF) measured by echocardiography or magnetic resonance imaging may not be sensitive enough to detect ultrastructural and physiologic improvements in cardiovascular function. For example, many patients with heart failure and severely impaired LVEF $(<30 \%)$ continue to lead active lives and demonstrate normal cardiac filling pressures with normal stroke volume. In similar fashion, preclinical and clinical studies of cell-based therapies should extend their evaluation of cardiac function beyond LVEF and begin to examine whether cell strategies impact hemodynamic function as measured by a conductance catheter in the pressure volume loop plane. Notably, a majority of preclinical studies also do not provide data regarding survival outcomes. Since many drug strategies improve survival, demonstrating an incremental benefit of cytotherapy over existing therapies in the heart failure population may prove challenging.

In the second review, Ye et al. examine strategies for tissue engineering after myocardial infarction by providing a comprehensive analysis of biomaterials and culture 
conditions used in animal models of myocardial infarction. Over the past two decades, more cardiovascular scientists are looking to the heart's own natural scaffold, namely, the extracellular matrix (ECM), for clues about how to best modulate cardiac structure and function. Cardiac ECM is composed primarily of types I $(80 \%)$ and III collagen $(10 \%)$. The remaining $10 \%$ is composed of types V and VI collagen, laminin, elastin, glycosaminoglycans, and proteoglycans. In the normal adult heart, individual cardiac myocytes are surrounded by a thin basement membrane composed of type IV collagen. Loosely interspersed among these individual myocytes are collagen fibrils, known as the endomysium. The perimysium organizes individual myocytes and myocardial capillaries into muscle bundles. The epimysium surrounds groups of muscle bundles and provides an additional layer of organization.

This natural collagen scaffold provides mechanical support to the myocardium, organizes muscle contraction, synthesizes and releases growth factors in a paracrine and autocrine fashion, and promotes wound healing. Excess collagen synthesis in response to myocardial stress exaggerates mechanical stiffness, disorganizes contraction due to myocyte separation, disrupts electrotonic connectivity, and worsens tissue hypoxia [5]. For these reasons, cardiac fibrosis can contribute to adverse cardiac remodeling in progressive heart failure and is an important target of therapy for these patients. More recently, studies have identified perivascular fibrosis as a distinct entity from interstitial fibrosis with a potentially important role as a scaffolding system closely integrated with the coronary vasculature [6].

The cells that generate this natural scaffold structure also have the ability to change phenotype. For example, cardiac fibroblasts can differentiate into a myofibroblast phenotype with the ability to contract and generate ECM [7]. Endothelial cells can also differentiate into various mesenchymal cell phenotypes in a process known as endothelialmesenchymal transition [8]. This critical step initially occurs during embryogenesis and contributes to cardiac remodeling in the adult heart after injury. Pericytes located in the adventitia of small and large vessels in the heart have also recently been identified as possible precursors to mesenchymal stem cells [9]. The potential of these various cell types as critical regulators of cardiac remodeling and regeneration is under active investigation.
Taken together, these findings suggest that tissue replacement strategies will require a better understanding of how the heart's natural scaffold and cellular components work together to maintain structural homeostasis in response to various stressors throughout life. Both review articles in this issue provide a comprehensive overview of the immense progress made to date and the promise of cytotherapeutics for patients with heart disease in the future.

\section{Disclosures None}

\section{References}

1. Rosamond, W., Flegal, K., Furie, K., Go, A., Greenlund, K., Haase, N., et al. (2008). Heart disease and stroke statistics-2008 update: a report from the American Heart Association Statistics Committee and Stroke Statistics Subcommittee. Circulation, 117(4), e25-e146.

2. Cowie, M. R., Mosterd, A., Wood, D. A., Deckers, J. W., Poole-Wilson, P. A., Sutton, G. C., et al. (1997). The epidemiology of heart failure. European Heart Journal, 18, 208-225.

3. Davies, M. K., Hobbs, F. D. R., Davis, R. C., Kenkre, J. E., Roalfe, A. K., Wosornu, D., et al. (2001). Prevalence of left ventricular systolic dysfunction and heart failure in the Echographic Heart of England Screening Study: a population based study. Lancet, 358, 439-444.

4. Slaughter, M. S., Rogers, J. G., Milano, C. A., Russell, S. D., Conte, J. V., Feldman, D., et al. (2009). Advanced heart failure treated with continuous-flow left ventricular assist device. The New England Journal of Medicine, 361, 2241-2251.

5. Berk, B. C., Fujiwara, K., \& Lehoux, S. (2007). ECM remodeling in hypertensive heart disease. The Journal of Clinical Investigation, $117(3), 568-575$.

6. Koitabashi, N., Danner, T., Zaiman, A. L., Pinto, Y. M., Rowell, J., \& Mankowski, J. (2011). Pivotal role of cardiomyocyte TGF- $\beta$ signaling in the murine pathological response to sustained pressure overload. Journal of Clinical Investigation, 121(6), 2301-2312.

7. Campbell, S. E., \& Katwa, L. C. (1997). Angiotensin II stimulated expression of transforming growth factor-betal in cardiac fibroblasts and myofibroblasts. Journal of Molecular and Cellular Cardiology, 29, 1947-1958.

8. Zeisberg, E. M., Tarnavski, O., Zeisberg, M., Dorfman, A. L., McMullen, J. R., Gustafsson, E., et al. (2007). Endothelialto-mesenchymal transition contributes to cardiac fibrosis. Nature Medicine, 13(8), 952-961.

9. Crisan, M., Corselli, M., Chen, C.W., Péault, B. (2011) Multilineage stem cells in the adult: A perivascular legacy? Organogenesis. 1;7(2). 